\title{
Residual ISI Obtained by Nonblind Adaptive Equalizers and Fractional Noise
}

\author{
Monika Pinchas \\ Department of Electrical and Electronic Engineering, Ariel University, 40700 Ariel, Israel \\ Correspondence should be addressed to Monika Pinchas; monika.pinchas@gmail.com
}

Received 18 July 2013; Accepted 29 August 2013

Academic Editor: Ming Li

Copyright (C) 2013 Monika Pinchas. This is an open access article distributed under the Creative Commons Attribution License, which permits unrestricted use, distribution, and reproduction in any medium, provided the original work is properly cited.

\begin{abstract}
Recently, a closed-form approximated expression was derived by the same author for the achievable residual intersymbol interference (ISI) case that depends on the step-size parameter, equalizer's tap length, input signal statistics, signal to noise ratio (SNR), and channel power and is valid for fractional Gaussian noise (fGn) input where the Hurst exponent is in the region of $0.5 \leq H<1$. But this expression was obtained for the blind adaptive case and cannot be applied to the nonblind adaptive version. Up to now, the achievable residual ISI for the non-blind adaptive case could be obtained only via simulation. In this paper, we derive a closed-form approximated expression (or an upper limit) for the residual ISI obtained by non-blind adaptive equalizers valid for fractional Gaussian noise (fGn) input where the Hurst exponent is in the region of $0.5 \leq H<1$. This new obtained expression depends on the step-size parameter, equalizer's tap length, input signal statistics, SNR, channel power, and the Hurst exponent parameter. Simulation results indicate that there is a high correlation between the calculated results (obtained from the new obtained expression for the residual ISI) and those obtained from simulating the system.
\end{abstract}

\section{Introduction}

We consider a nonblind deconvolution problem in which we observe the output of an unknown, possibly nonminimum phase, linear system (single-input-single-output (SISO) FIR system) from which we want to recover its input (source) using an adjustable linear filter (equalizer) and training symbols. During transmission, a source signal undergoes a convolutive distortion between its symbols and the channel impulse response. This distortion is referred to as ISI [1, 2]. It is well known that ISI is a limiting factor in many communication environments where it causes an irreducible degradation of the bit error rate thus imposing an upper limit on the data symbol rate. In order to overcome the ISI problem, an equalizer is implemented in those systems [1-12].

In this paper, we consider the nonblind adaptive equalizer where training sequences are needed to generate the error that is fed into the adaptive mechanism which updates the equalizer's taps [9-12]. The nonblind adaptive approach yields in most cases a better equalization performance considering convergence speed and equalization quality compared with the blind adaptive version [6]. In addition, the blind adaptive version has a higher computational cost compared with its nonblind approach [6].

The equalization performance from the residual ISI point of view depends on the channel characteristics, on the added noise, on the step-size parameter used in the adaptation process, on the equalizer's tap length and on the input signal statistics $[13,14]$. Fast convergence speed and reaching a residual ISI where the eye diagram is considered to be open (for the communication case) are the main requirements from a blind or nonblind equalizer. Fast convergence speed may be obtained by increasing the step-size parameter. But, increasing the step-size parameter may lead to a higher residual ISI which might not meet the system's requirements any more. Recently [2], a closed-form approximated expression was derived for the achievable residual ISI case that depends on the step-size parameter, equalizer's tap length, input signal statistics, SNR, Hurst exponent, and channel power. But this expression is valid only for the blind adaptive case and cannot be used for the nonblind version.

Up to now, the achievable residual ISI for the nonblind adaptive case (for the noisy or noiseless case) could be obtained only via simulation. Thus, the system designer had 
to spend a lot of time in simulating the whole system in order to find the best values for the step-size parameter and equalizer's tap length that meet the system's requirements from the residual ISI point of view. In this paper, we derive a closed-form approximated expression (or an upper limit) for the residual ISI obtained by nonblind adaptive equalizers that depends on the step-size parameter, equalizer's tap length, input signal statistics, SNR, channel power, and Hurst exponent parameter. This expression is valid for fGn input where the Hurst exponent is in the region of $0.5 \leq H<1$. Please note, $H=1$ is the limit case, which does not have much practical sense [15-17]. It should be pointed out that a white Gaussian process is a special case $(H=0.5)$ of the fractional Gaussian noise (fGn) model [18]. FGn with $H \in(0.5,1)$ corresponds to the case of long-range dependency (LRD) [18]. Thus, the new obtained expression for the achievable residual ISI is not only valid for the special case of white Gaussian process but also covers those cases that correspond to the case of LRD.

The paper is organized as follows. After having described the system under consideration in Section 2, the closed-form approximated expression (or upper limit) for the residual ISI is introduced in Section 3. In Section 4, simulation results are presented, and the conclusion is given in Section 5.

\section{System Description}

The system under consideration is illustrated in Figure 1, where we make the following assumptions.

(1) The input sequence $x[n]$ belongs to a two independent quadrature carriers case constellation input with variance $\sigma_{x}^{2}$, where $x_{r}[n]$ and $x_{i}[n]$ are the real and imaginary parts of $x[n]$, respectively, and $\sigma_{x_{r}}^{2}$ is the variance of $x_{r}[n]$.

(2) The unknown channel $h[n]$ is a possibly nonminimum phase linear time-invariant filter in which the transfer function has no "deep zeros"; namely, the zeros lie sufficiently far from the unit circle.

(3) The equalizer $c[n]$ is a tap-delay line.

(4) The noise $w[n]$ consists of $w[n]=w_{r}[n]+j w_{i}[n]$, where $w_{r}[n]$ and $w_{i}[n]$ are the real and imaginary parts of $w[n]$, respectively, and $w_{r}[n]$ and $w_{i}[n]$ are independent. Both $w_{r}[n]$ and $w_{i}[n]$ are fractional Gaussian noises (fGn) with zero mean. Note that $\sigma_{w_{r}}^{2}=E\left[w_{r}^{2}[n]\right], \sigma_{w_{i}}^{2}=E\left[w_{i}^{2}[n]\right]$, for $m \neq k$ : $E\left[w_{r}[n-k] w_{r}[n-m]\right]=\left(\sigma_{w_{r}}^{2} / 2\right)\left[(|m-k|-1)^{2 H}-\right.$ $\left.2(|m-k|)^{2 H}+(|m-k|+1)^{2 H}\right]$ and $E\left[w_{i}[n-k] w_{i}[n-\right.$ $m]]=\left(\sigma_{w_{i}}^{2} / 2\right)\left[(|m-k|-1)^{2 H}-2(|m-k|)^{2 H}+\right.$ $\left.(|m-k|+1)^{2 H}\right]$, where $E[\cdot]$ denotes the expectation operator on $(\cdot)$ and $H$ is the Hurst exponent.

(5) The variance of $w[n]$ is denoted as $E\left[w[n] w^{*}[n]\right]=$ $\sigma_{w}^{2}$, where $\sigma_{w}^{2}=2 \sigma_{w_{i}}^{2}=2 \sigma_{w_{r}}^{2}$ and $(\cdot)^{*}$ is the conjugate operation on $(\cdot)$.

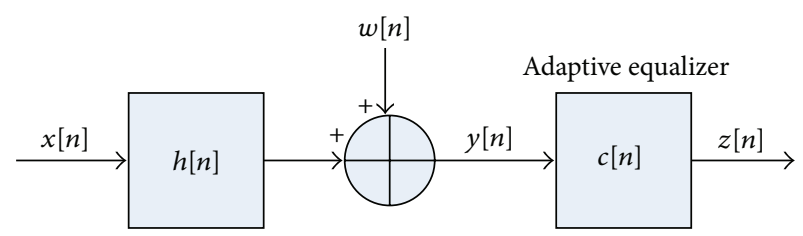

FIGURE 1: Block diagram of a baseband communication system.

The transmitted sequence $x[n]$ is sent through the channel $h[n]$ and is corrupted with noise $w[n]$. Therefore, the equalizer's input sequence $y[n]$ may be written as

$$
y[n]=x[n] * h[n]+w[n],
$$

where “*” denotes the convolution operation. The equalized output signal can be written as

$$
z[n]=x[n]+p[n]+\widetilde{w}[n],
$$

where $p[n]$ is the convolutional noise, namely, the residual intersymbol interference (ISI) arising from the difference between the ideal equalizer's coefficients and those chosen in the system and $\widetilde{w}[n]=w[n] * c[n]$. The ISI is often used as a measure of performance in equalizers' applications, defined by

$$
\text { ISI }=\frac{\sum_{\widetilde{m}}\left|\widetilde{s}_{\widetilde{m}}\right|^{2}-|\widetilde{s}|_{\text {max }}^{2}}{\mid \tilde{s}_{\max }^{2}},
$$

where $|\widetilde{s}|_{\max }$ is the component of $\widetilde{s}$, given in (4), having the maximal absolute value. Consider that

$$
\widetilde{s}[n]=c[n] * h[n]=\delta[n]+\zeta[n],
$$

where $\delta$ is the Kronecker delta function and $\zeta[n]$ stands for the difference (error) between the ideal and the actual value used for $c[n]$.

Next, we turn to the adaptation mechanism of the equalizer which is based on training symbols $[9-12,19]$ :

$$
\underline{c}_{\mathrm{eq}}[n+1]=\underline{c}_{\mathrm{eq}}[n]-\mu(z[n]-x[n]) \underline{y}^{*}[n],
$$

where $\mu$ is the step-size parameter, $\underline{c}_{\mathrm{eq}}[n]$ is the equalizer vector where the input vector is $y[n]=[y[n] \cdots y[n-N+1]]^{T}$, and $N$ is the equalizer's tap length. The operator ()$^{T}$ denotes for transpose of the function (). Please note that for the nonblind adaptive case, during the training period, a known data sequence is transmitted. A replica of this sequence is made available at the receiver in proper synchronism with the transmitter, thereby making it possible for adjustments to be made to the equalizer coefficients in accordance with the adaptive filtering algorithm employed in the equalizer design [19].

\section{Residual ISI for Fractional Gaussian Noise Input}

In this section, a closed-form approximated expression (or an upper limit) is derived for the residual ISI valid for the fGn input case. 
Theorem 1. Noted the following assumptions.

(1) The convolutional noise $p[n]$ is a zero mean, white Gaussian process with variance $\sigma_{p}^{2}=E\left[p[n] p^{*}[n]\right]$. The real part of $p[n]$ is denoted as $p_{r}[n]$ and $E\left[p_{r}^{2}[n]\right]=$ $m_{p}$.

(2) The source signal $x[n]$ is a rectangular Quadrature Amplitude Modulation (QAM) signal (where the real part of $x[n]$ is independent of the imaginary part of $x[n])$ with known variance and higher moments.

(3) The convolutional noise $p[n]$ and the source signal are independent.

(4) The gain between the source and equalized output signal is equal to one.

(5) The convolutional noise $p[n]$ is independent of $\widetilde{w}[n]$.

(6) The added noise is $f G n$ with zero mean.

(7) The channel $h[n]$ has real coefficients.

(8) The Hurst exponent is in the range of $0.5 \leq H<1$.

The residual ISI expressed in $\mathrm{dB}$ units may be defined as

$$
\text { ISI }=10 \log _{10}\left(m_{p}\right)-10 \log _{10}\left(\sigma_{x_{r}}^{2}\right),
$$

where $m_{p}$ is defined by

$$
\begin{gathered}
m_{p}=\sigma_{\widetilde{w}_{r}}^{2}\left(\mu\left(N \sigma_{x}^{2} \sum_{k=0}^{k=R-1} h_{k}^{2}[n]+\frac{N \sigma_{x}^{2}}{\mathrm{SNR}}\right)^{2}\right. \\
\times\left(2\left(N \sigma_{x}^{2} \sum_{k=0}^{k=R-1} h_{k}^{2}[n]+\frac{N \sigma_{x}^{2}}{\mathrm{SNR}}\right)\right. \\
\left.\left.-\mu\left(N \sigma_{x}^{2} \sum_{k=0}^{k=R-1} h_{k}^{2}[n]+\frac{N \sigma_{x}^{2}}{\mathrm{SNR}}\right)^{2}\right)^{-1}\right) \\
=\sigma_{\widetilde{w}_{r}}^{2} \frac{\mu\left(N \sigma_{x}^{2} \sum_{k=0}^{k=R-1} h_{k}^{2}[n]+\left(N \sigma_{x}^{2} / \mathrm{SNR}\right)\right)}{2-\mu\left(N \sigma_{x}^{2} \sum_{k=0}^{k=R-1} h_{k}^{2}[n]+\left(N \sigma_{x}^{2} / \mathrm{SNR}\right)\right)} \\
\sigma_{\widetilde{w}_{r}}^{2} \cong \frac{\sigma_{x_{r}}^{2}}{\operatorname{SNR} \sum_{k=0}^{k=R-1} h_{k}^{2}[n]}[1+\sqrt{(N-1)} H(2 H-1)]
\end{gathered}
$$

and $R$ is the channel length, $\sigma_{\widetilde{w}_{r}}^{2}$ is the variance of $\widetilde{w}_{r}[n]\left(\widetilde{w}_{r}[n]\right.$ is the real part of $\widetilde{w}[n])$, and SNR is given by SNR $=\sigma_{x_{r}}^{2} / \sigma_{w_{r}}^{2}=$ $\sigma_{x}^{2} / \sigma_{w}^{2}$

Comments. It should be pointed out that assumptions (1)-(5) from above are precisely the same assumptions made in [2, 14].

Proof. Let us first recall the expression for the adaptation mechanism of the equalizer given in (5). Then, we substitute (2) into (5) and obtain

$$
\underline{c}_{\mathrm{eq}}[n+1]=\underline{c}_{\mathrm{eq}}[n]-\mu(p[n]+\widetilde{w}[n]) \underline{y}^{*}[n] .
$$

Next, we recall from [14] the expression for $E\left[\Delta\left(p_{r}^{2}\right)\right]$, where $p_{r}$ is the real part of $p[n]$ and $\Delta\left(p_{r}^{2}\right)=p_{r}^{2}[n+1]-p_{r}^{2}[n]$ :

$$
\begin{aligned}
& E\left[\Delta\left(p_{r}^{2}\right)\right] \\
& \cong-2 E\left[\operatorname{Pr}\left(\mu P_{r}(z) \sum_{m=0}^{m=N-1} y[n-m] y^{*}[n-m]\right)\right] \\
& +E\left[\left(-\mu P_{r}(z) \sum_{m=0}^{m=N-1} y[n-m] y^{*}[n-m]\right)^{2}\right],
\end{aligned}
$$

where $P_{r}(z)$ is the real part of $P(z)$ and is given in our case as $P(z)=z[n]-x[n]=p[n]+\widetilde{w}[n] \Longrightarrow P_{r}(z)=p_{r}+\widetilde{w}_{r}[n]$.

According to $[13,14]$, when the equalizer has converged, we may assume that $E\left[\Delta\left(p_{r}^{2}\right)\right] \cong 0$. Therefore, by setting $E\left[\Delta\left(p_{r}^{2}\right)\right]=0$ into (9), we obtain

$$
\begin{aligned}
& -2 \mu m_{p} E\left[\sum_{m=0}^{m=N-1} y[n-m] y^{*}[n-m]\right] \\
& +\mu^{2}\left(m_{p}+\sigma_{\widetilde{w}_{r}}^{2}\right) \\
& \times E\left[\left(\sum_{m=0}^{m=N-1} y[n-m] y^{*}[n-m]\right)^{2}\right]=0 \\
& \Downarrow \\
& m_{p}=\sigma_{\widetilde{w}_{r}}^{2}\left(\mu E\left[\left(\sum_{m=0}^{m=N-1} y[n-m] y^{*}[n-m]\right)^{2}\right]\right. \\
& \times\left(2 E\left[\sum_{m=0}^{m=N-1} y[n-m] y^{*}[n-m]\right]-\mu E\right. \\
& \left.\left.\times\left[\left(\sum_{m=0}^{m=N-1} y[n-m] y^{*}[n-m]\right)^{2}\right]\right)^{-1}\right) .
\end{aligned}
$$

In [13], the expression $E\left[\left(\sum_{m=0}^{m=N-1} y[n-m] y^{*}[n-m]\right)^{2}\right]$ was approximated as $\left(E\left[\sum_{m=0}^{m=N-1} y[n-m] y^{*}[n-m]\right]\right)^{2}$. It should be pointed out that this approximation fits the MPSK case where QPSK is a special case of it. However, satisfying results were obtained in [13] for the 16QAM and 64QAM cases in spite of the fact that the above mentioned approximation was applied. Thus, it makes sense to use the same approximation also here for our case. The expression $E\left[\sum_{m=0}^{m=N-1} y[n-m] y^{*}[n-m]\right]$ may be written as

$$
E\left[\sum_{m=0}^{m=N-1} y[n-m] y^{*}[n-m]\right]=N \sigma_{x}^{2} \sum_{k=0}^{k=R-1} h_{k}^{2}[n]+\frac{N \sigma_{x}^{2}}{\mathrm{SNR}} .
$$




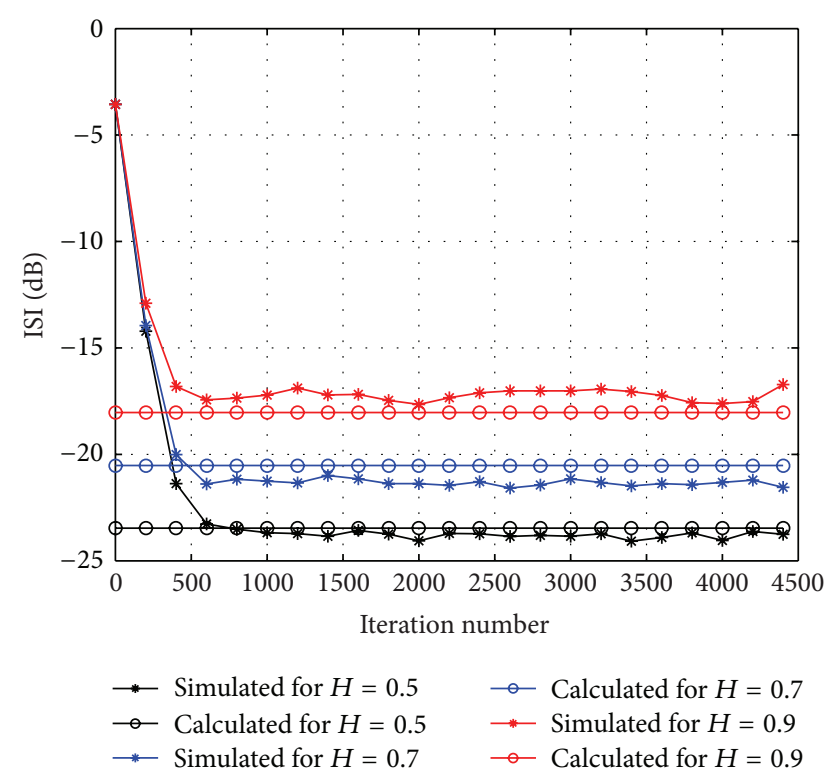

FIGURE 2: A comparison between the simulated and calculated residual ISI for the 16QAM source input going through channell for SNR $=10[\mathrm{~dB}]$. The averaged results were obtained in 100 Monte Carlo trials. The equalizer's tap length and step-size parameter were set to 13 and 0.0006 respectively.

Next, we turn to find a closed-form approximated expression for $\sigma_{\widetilde{w}_{r}}^{2}$. The real part of $\widetilde{w}[n]$, namely, $\widetilde{w}_{r}[n]$, may be expressed as

$$
\widetilde{w}_{r}[n]=\sum_{k=0}^{k=N-1} c_{k}[n] w_{r}[n-k]
$$

Thus, the variance of $\widetilde{w}_{r}[n]$ is given by:

$$
\begin{aligned}
\sigma_{\widetilde{w}_{r}}^{2} & =E\left[\sum_{k=0}^{k=N-1} c_{k}[n] w_{r}[n-k] \sum_{m=0}^{m=N-1} c_{m}[n] w_{r}[n-m]\right] \\
& =\sum_{k=0}^{k=N} \sum_{m=0}^{m=N-1} c_{k}[n] c_{m}[n] E\left[w_{r}[n-k] w_{r}[n-m]\right],
\end{aligned}
$$

which can be also written as

$$
\begin{aligned}
\sigma_{\widetilde{w}_{r}}^{2}= & \sigma_{w_{r}}^{2} \sum_{k=0}^{k=N-1} c_{k}^{2}[n] \\
& +\sum_{k=0, k \neq m}^{k=N-1} \sum_{m=0, k \neq m}^{m=N-1} c_{k}[n] c_{m}[n] E\left[w_{r}[n-k] w_{r}[n-m]\right] .
\end{aligned}
$$

According to [2], expression (15) can be approximately written as

$$
\sigma_{\widetilde{w}_{r}}^{2} \cong \frac{\sigma_{x_{r}}^{2}}{\operatorname{SNR} \sum_{k=0}^{k=R-1} h_{k}^{2}[n]}[1+\sqrt{N-1} H(2 H-1)],
$$

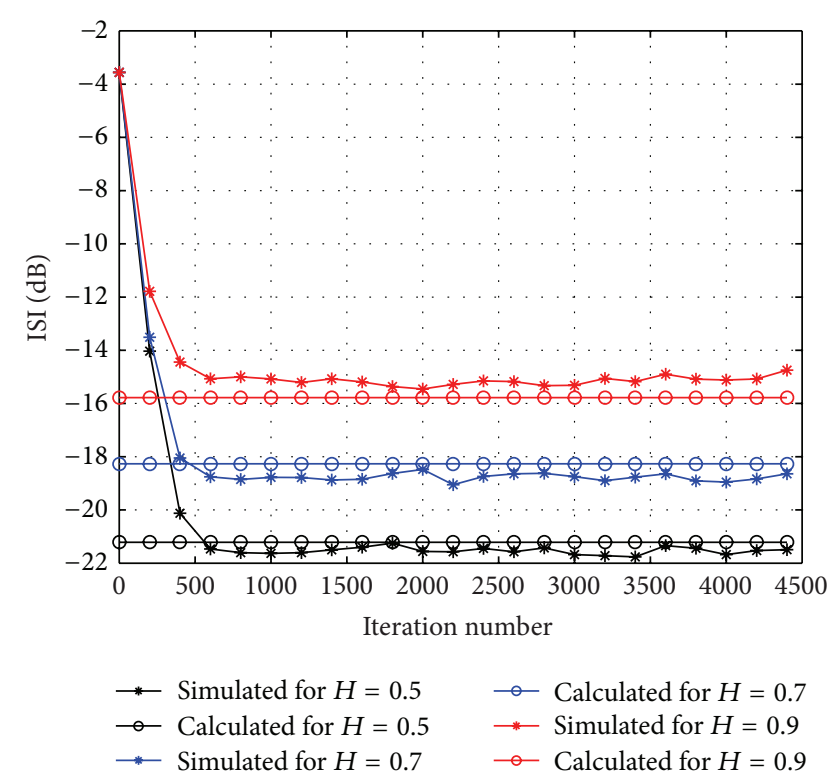

FIgURE 3: A comparison between the simulated and calculated residual ISI for the 16QAM source input going through channell for SNR $=8[\mathrm{~dB}]$. The averaged results were obtained in 100 Monte Carlo trials. The equalizer's tap length and step-size parameter were set to 13 and 0.0006 respectively.

by using assumption (4) from the system description section, assumptions (4) and (6)-(8) from this section, the Holder inequality [20], and the following approximation [21]:

$$
\begin{aligned}
& 0.5\left[(|m-k|-1)^{2 H}-2(|m-k|)^{2 H}+(|m-k|+1)^{2 H}\right] \\
& \simeq H(2 H-1)|m-k|^{2 H-2} .
\end{aligned}
$$

Now, by substituting (12) and (16) into (11) we obtain (7). This completes our proof.

\section{Simulation}

In this section, we test our new proposed expression for the residual ISI for the 16QAM case (a modulation using $\pm\{1,3\}$ levels for in-phase and quadrature components) with the algorithm described in (5) for different SNR, step-size, and equalizer's tap length values and for two different channel types. The following two channels were considered. Channell (initial ISI = 0.44): the channel parameters were determined according to [22]

$$
\begin{array}{r}
h_{n}=(0 \text { for } n<0 ;-0.4 \text { for } n=0 \\
\left.\quad \times 0.84 \cdot 0.4^{n-1} \text { for } n>0\right) .
\end{array}
$$

Channel2 (initial ISI $=0.88$ ) the channel parameters were determined according to

$$
h_{n}=(0.4851,-0.72765,-0.4851) \text {. }
$$

The equalizer was initialized by setting the center tap equal to one and all others to zero. 


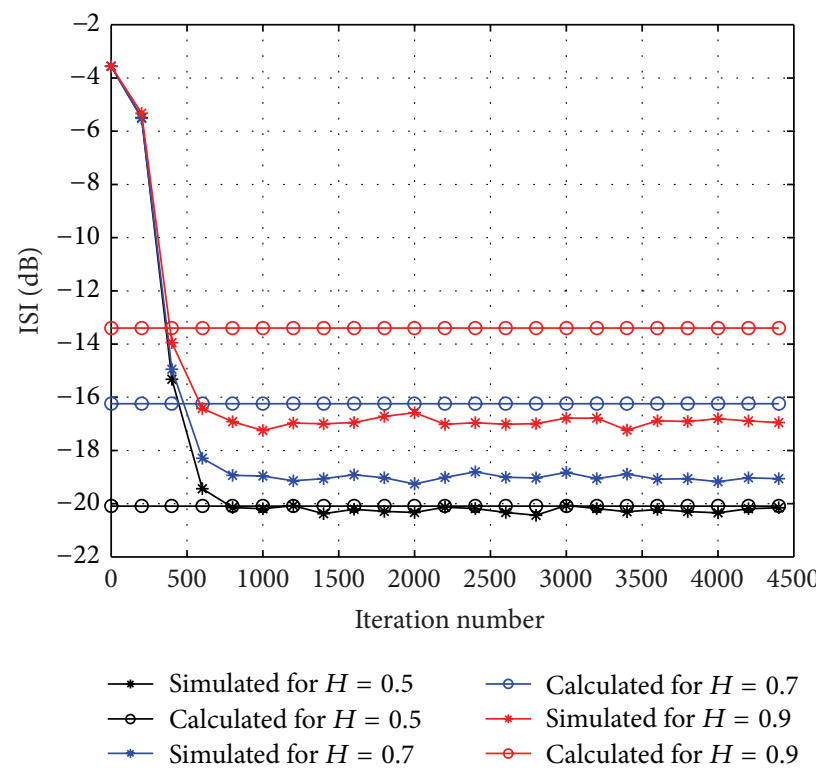

FIGURE 4: A comparison between the simulated and calculated residual ISI for the 16QAM source input going through channel1 for SNR $=10$ $[\mathrm{dB}]$. The averaged results were obtained in 100 Monte Carlo trials. The equalizer's tap length and step-size parameter were set to 27 and 0.0006 respectively.

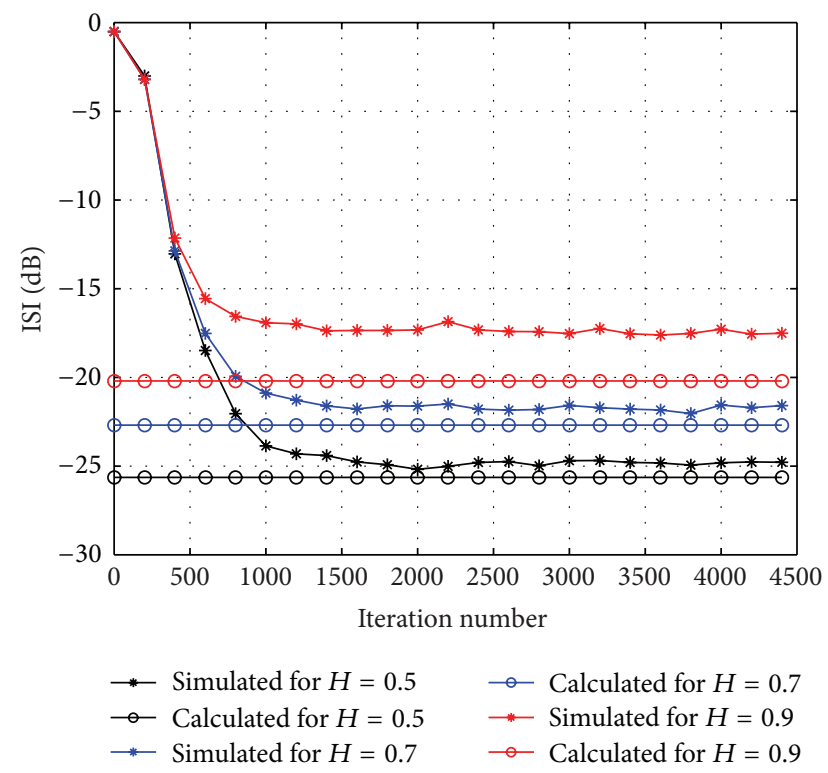

FIgURE 5: A comparison between the simulated and calculated residual ISI for the 16QAM source input going through channel2 for SNR $=12[\mathrm{~dB}]$. The averaged results were obtained in 100 Monte Carlo trials. The equalizer's tap length and step-size parameter were set to 13 and 0.0006 respectively.

In the following, we denote the residual ISI performance according to (6) with (7) as "Calculated ISI." Figure 2 to Figure 8 show the ISI performance as a function of the iteration number of our proposed expression (6) with (7) for the achievable residual ISI compared with the simulated results for two different channels and equalizer's tap length

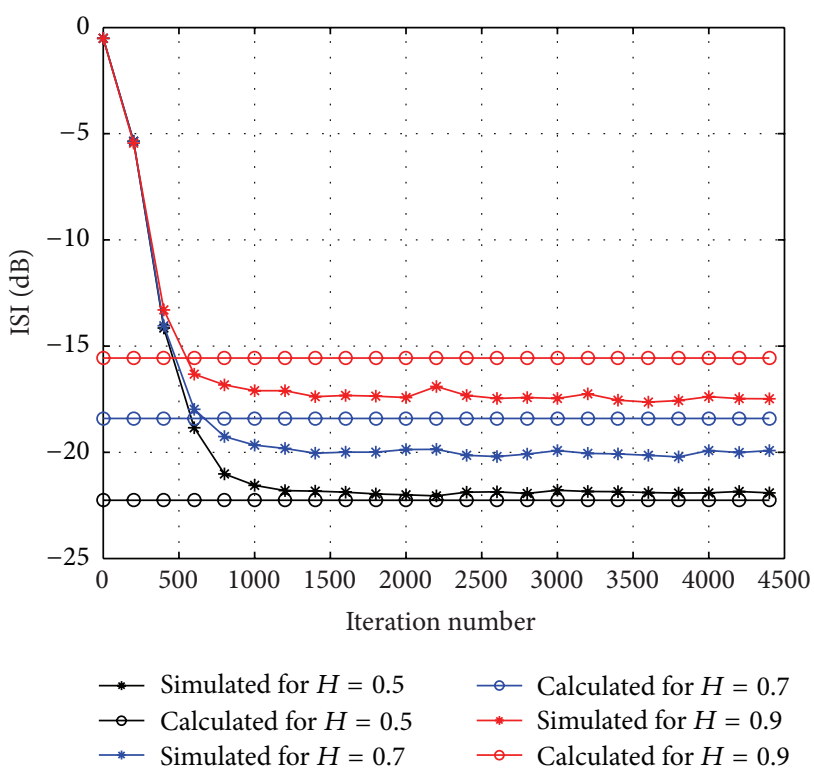

Figure 6: A comparison between the simulated and calculated residual ISI for the 16QAM source input going through channel2 for SNR $=12[\mathrm{~dB}]$. The averaged results were obtained in 100 Monte Carlo trials. The equalizer's tap length and step-size parameter were set to 27 and 0.0006 respectively.

and various values for $H, \mathrm{SNR}$ and step-size parameter. According to Figures 2, 3, 5, 6, 7, and 8, a high correlation is observed between the simulated and calculated results even for $H=0.9$. According to Figure 4, the calculated ISI may be considered as an upper limit for the simulated results for $H>0.5$. 


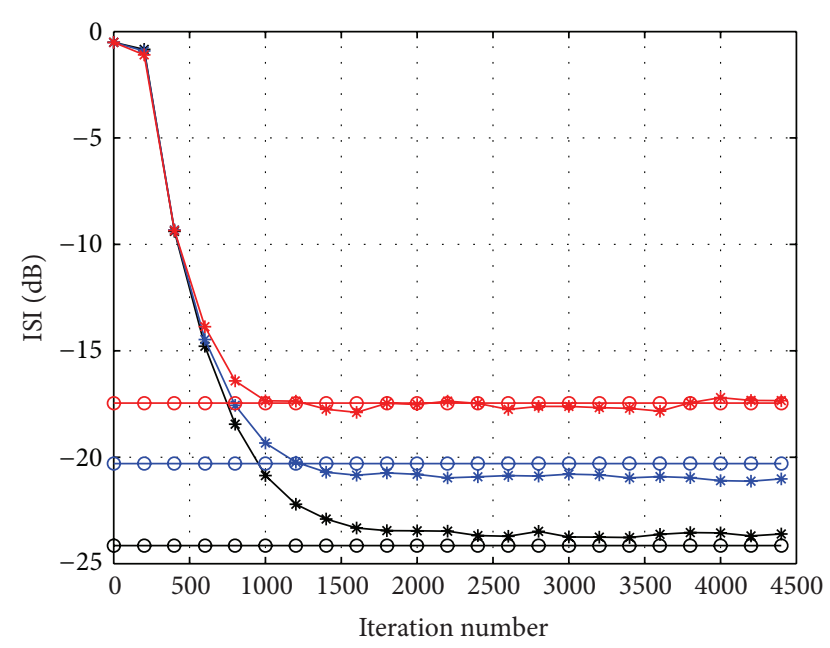

FIgURE 7: A comparison between the simulated and calculated residual ISI for the 16QAM source input going through channel2 for $\mathrm{SNR}=12[\mathrm{~dB}]$. The averaged results were obtained in 100 Monte Carlo trials. The equalizer's tap length and step-size parameter were set to 27 and 0.0004 respectively.

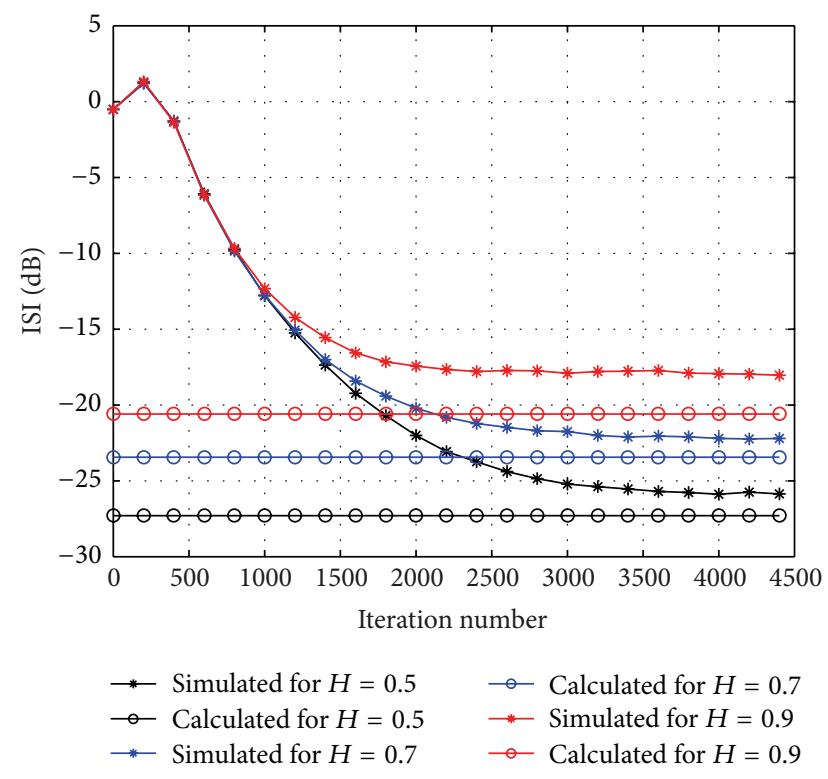

FIGURE 8: A comparison between the simulated and calculated residual ISI for the 16QAM source input going through channel2 for $\mathrm{SNR}=12[\mathrm{~dB}]$. The averaged results were obtained in 100 Monte Carlo trials. The equalizer's tap length and step-size parameter were set to 27 and 0.0002 respectively.

\section{Conclusion}

In this paper, we proposed a closed-form approximated expression (or an upper limit) for the residual ISI obtained by nonblind adaptive equalizers valid for the fGn input case where the Hurst exponent is in the region of $0.5 \leq H<$ 1. This new obtained expression depends on the step-size parameter, equalizer's tap length, input signal statistics, SNR, channel power, and the Hurst exponent parameter. According to simulation results, a high correlation is obtained between the calculated and simulated results for the residual ISI for some cases, while for others the new obtained expression is a relative tight upper limit for the averaged residual ISI results.

\section{References}

[1] M. Pinchas, "Two blind adaptive equalizers connected in series for equalization performance improvement," Journal of Signal and Information Processing, vol. 4, no. 1, pp. 64-71, 2013.

[2] M. Pinchas, "Residual ISI obtained by blind adaptive equalizers and fractional noise," Mathematical Problems in Engineering, vol. 2013, Article ID 972174, 11 pages, 2013.

[3] M. Pinchas and B. Z. Bobrovsky, "A maximum entropy approach for blind deconvolution," Signal Processing, vol. 86, no. 10, pp. 2913-2931, 2006.

[4] M. Pinchas and B. Z. Bobrovsky, "A novel HOS approach for blind channel equalization," IEEE Transactions on Wireless Communications, vol. 6, no. 3, pp. 875-886, 2007.

[5] M. Pinchas, Blind Equalizers by Techniques of Optimal NonLinear Filtering Theory, VDM Verlagsservice Gesellschaft mbH, 2009.

[6] M. Pinchas, The Whole Story Behind Blind Adaptive Equalizers/Blind Deconvolution, Bentham Science Publishers, 2012.

[7] G. H. Im, C. J. Park, and H. C. Won, "A blind equalization with the sign algorithm for broadband access," IEEE Communications Letters, vol. 5, no. 2, pp. 70-72, 2001.

[8] D. N. Godard, "Self recovering equalization and carrier tracking in two-dimenional data communication system," IEEE Transactions on Communications Systems, vol. 28, no. 11, pp. 1867-1875, 1980.

[9] M. Reuter and J. R. Zeidler, "Nonlinear effects in LMS adaptive equalizers," IEEE Transactions on Signal Processing, vol. 47, no. 6, pp. 1570-1579, 1999.

[10] A. H. I. Makki, A. K. Dey, and M. A. Khan, "Comparative study on LMS and CMA channel equalization," in Proceedings of the International Conference on Information Society (i-Society '10), pp. 487-489, June 2010.

[11] E. Tucu, F. Akir, and A. Zen, "New step size control technique for blind and non-blind equalization algorithms," Radioengineering, vol. 22, no. 1, p. 44, 2013.

[12] http://www.academypublisher.com/proc/iscsct10/papers/iscsct10p256.pdf.

[13] M. Pinchas, "A closed approximated formed expression for the achievable residual intersymbol interference obtained by blind equalizers," Signal Processing, vol. 90, no. 6, pp. 1940-1962, 2010.

[14] M. Pinchas, "A new closed approximated formed expression for the achievable residual ISI obtained by adaptive blind equalizers for the noisy case," in Proceedings of the IEEE International Conference on Wireless Communications, Networking and Information Security (WCNIS '10), pp. 26-30, Beijing, China, June 2010.

[15] J. Beran, Statistics for Long-Memory Processes, vol. 61 of Monographs on Statistics and Applied Probability, Chapman and Hall, New York, NY, USA, 1994.

[16] M. Li and W. Zhao, “On 1/ $f$ noise," Mathematical Problems in Engineering, vol. 2012, Article ID 673648, 23 pages, 2012.

[17] M. Li, "Fractal time series-a tutorial review," Mathematical Problems in Engineering, vol. 2010, Article ID 157264, 26 pages, 2010. 
[18] M. Li and W. Zhao, "Quantitatively investigating locally weak stationarity of modified multifractional Gaussian noise," Physica A, vol. 391, no. 24, pp. 6268-6278, 2012.

[19] G. Malik and A. S. Sappal, "Adaptive equalization algorithms: an overview," International Journal of Advanced Computer Science and Applications, vol. 2, no. 3, 2011.

[20] M. R. Spiegel, Mathematical Handbook of Formulas and Tables, Schaum's Outline Series, McGraw-Hill, New York, NY, USA, 1968.

[21] M. Li and W. Zhao, "On bandlimitedness and lag-limitedness of fractional Gaussian noise," Physica A, vol. 392, no. 9, pp. 19551961, 2013.

[22] O. Shalvi and E. Weinstein, "New criteria for blind deconvolution of nonminimum phase systems (channels)," IEEE Transactions on Information Theory, vol. 36, no. 2, pp. 312-321, 1990. 


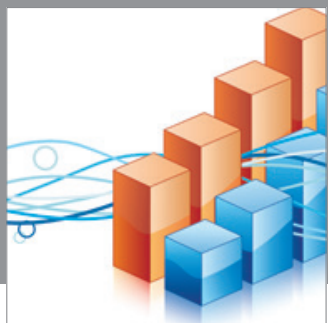

Advances in

Operations Research

mansans

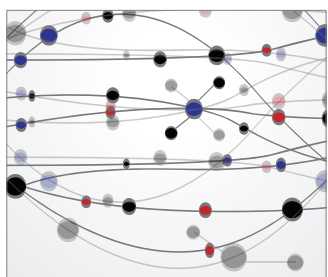

The Scientific World Journal
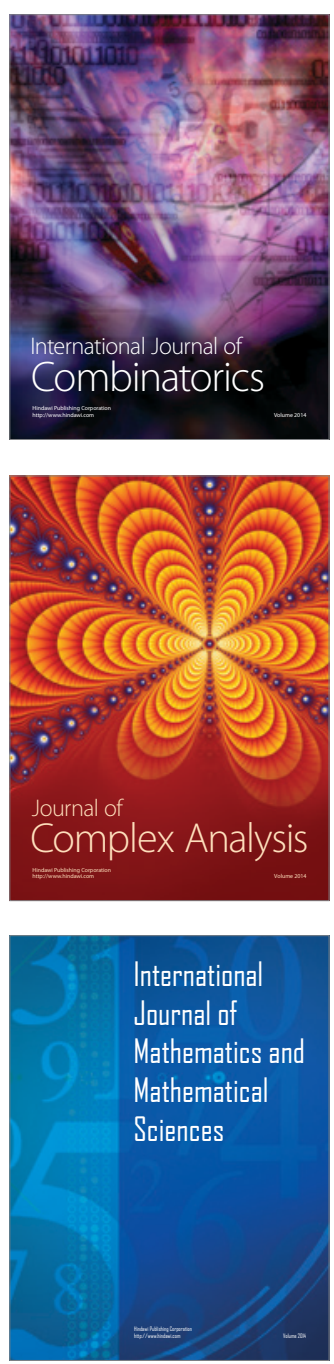
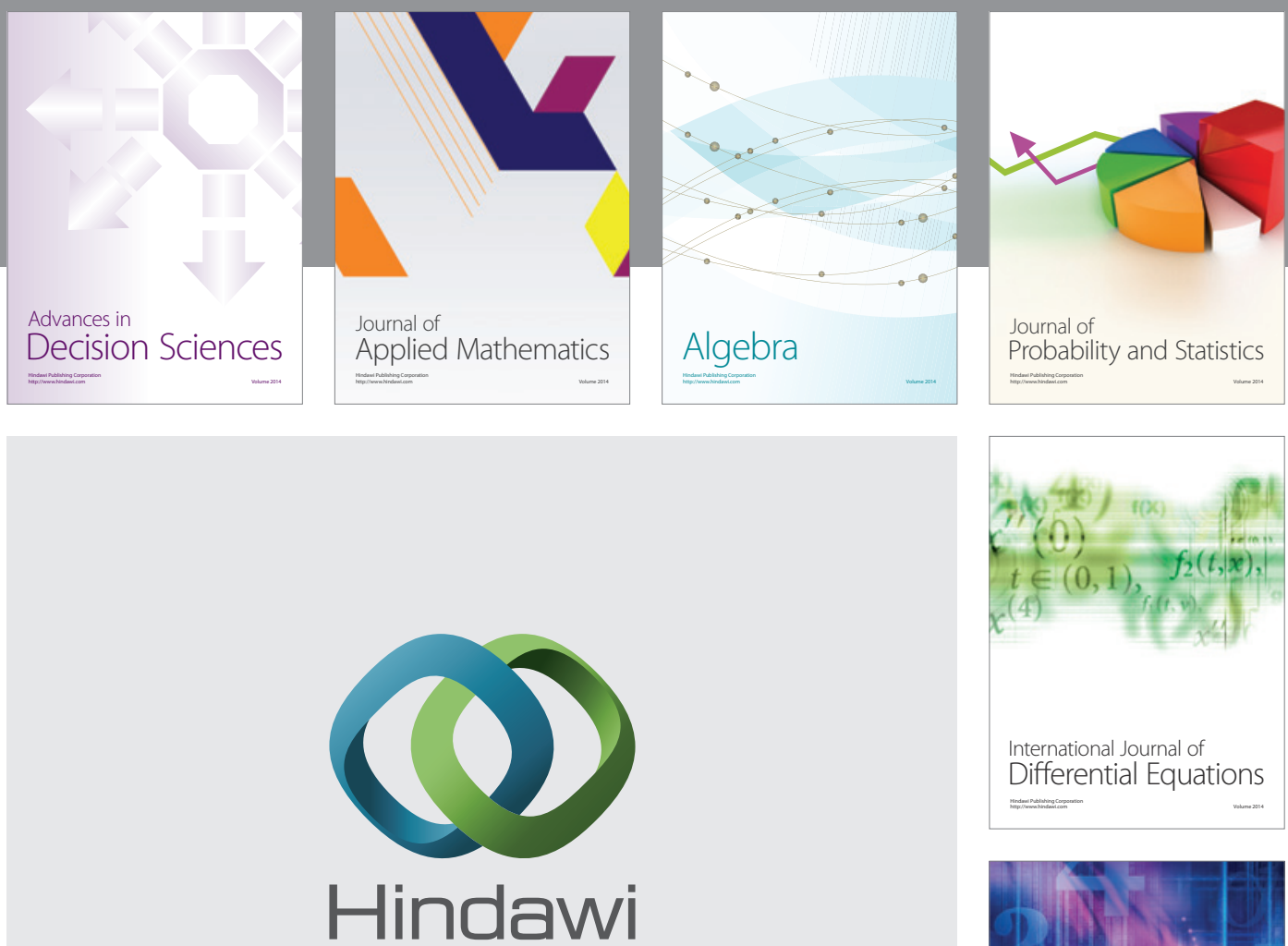

Submit your manuscripts at http://www.hindawi.com
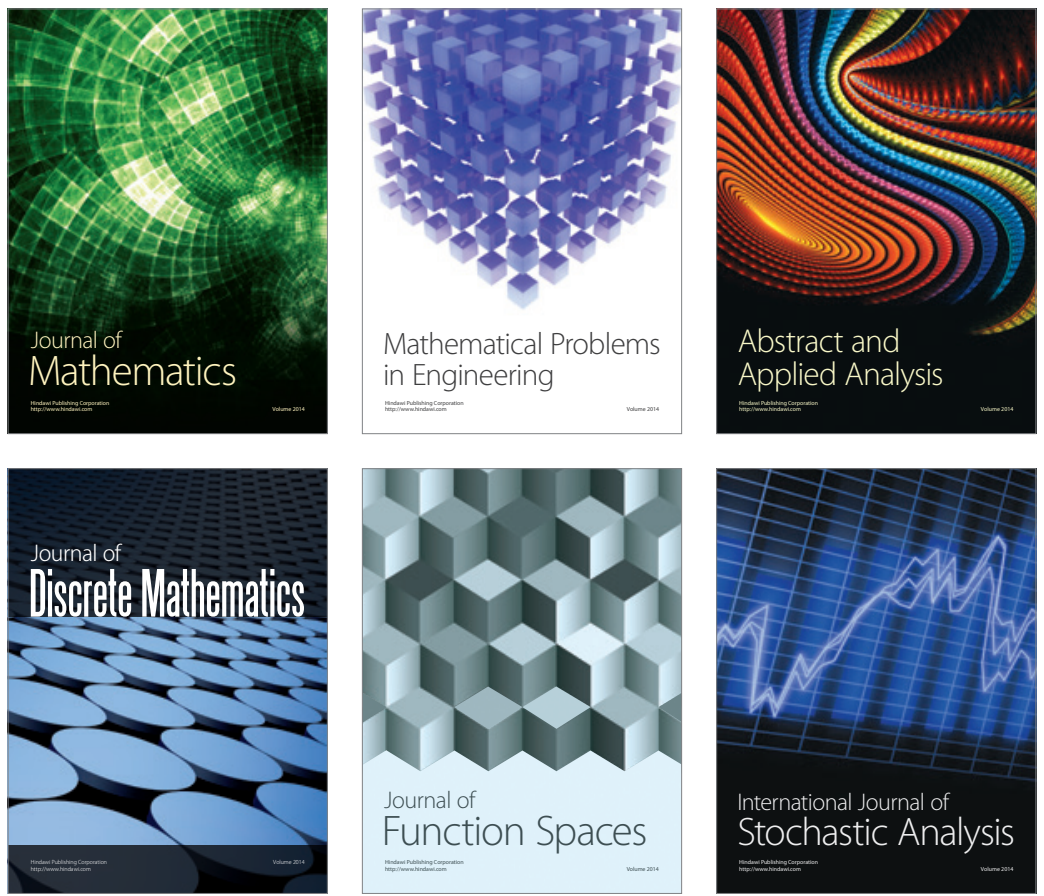

Journal of

Function Spaces

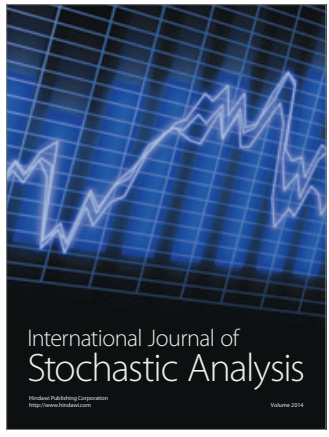

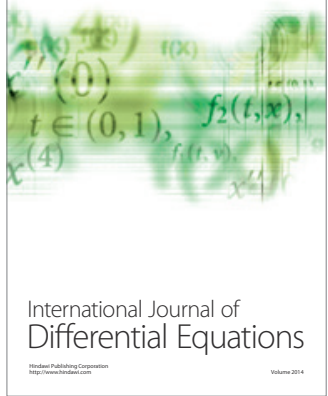
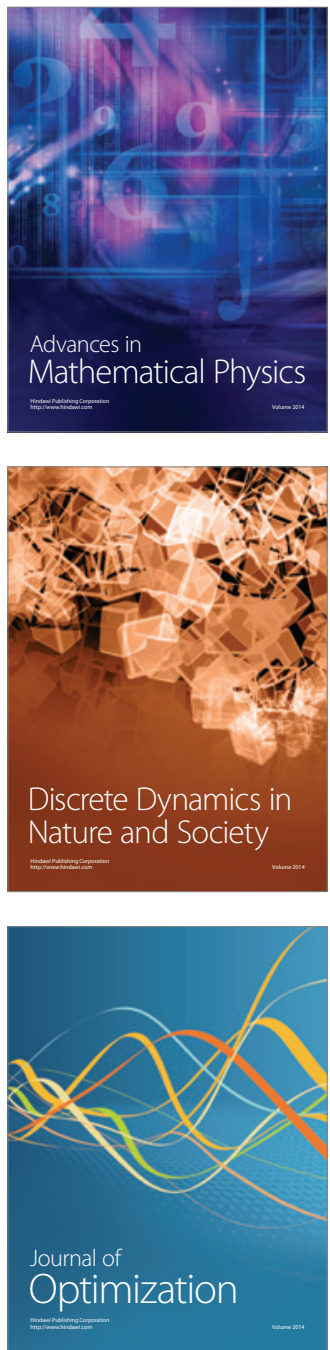